\title{
LEVANTAMENTO EPIDEMIOLÓGICO DO HIV/AIDS NO MUNICÍPIO DE PARNAÍBA-PI, BRASIL, 1990-2018
}

Epidemiological survey of HIV/AIDS in the municipality of ParnaíbaPI, Brazil, 1990-2018

Encuesta epidemiológica del VIH/SIDA en el municipio de Parnaíba-PI, Brasil, 1990-2018

Francisco Irisvan Coelho de Resende Dias • Discente do Curso de Fisioterapia da Universidade Federal do Piauí-UFPI • E-mail: irisvancoelho13@gmail.com

Cláudia Lima Mascarenhas Diniz • Discente do Curso de Medicina da Universidade Federal do Piauí-UFPI • E-mail: mascarenhas@gmail.com

Rafael Sambuichi • Discente do Curso de Medicina da Universidade Federal do Piauí-UFPI • E-mail: rafaelsambuichi@gmail.com

Lucélia Soares da Silva • Enfermeira na Secretaria Municipal de Saúde, Parnaíba-PI - Especialista em Formação Integrada Multiprofissional em Educação Permanente em Saúde-UFRGS • E-mail: lucelia09@hotmai 1.com

Francisco Jander de Sousa Nogueira • Professor da Universidade Federal do PiauíUFPI • Doutor em Sociologia-UFPB • E-mail: jander.sociosaude@gmail.com

Cláudio Ângelo Ventura • Professor da Universidade Federal do Piaúi-UFPI • Doutor em Biologia Funcional e Molecular-UNICAMP •

E-mail: claudioangelo09@gmail.com

Autor responsável pela correspondência:

Cláudio Ângelo Ventura • E-mail: claudioangelo09@gmail.com 


\section{ciêncíncia
pural}

\section{RESUMO}

Introdução: A epidemia do HIV/AIDS é um desafio para saúde pública mundial e leva ao óbito milhares de pessoas todos os anos. Hoje é considerada uma condição crônica, com mudanças epidemiológicas importantes que necessitam ser conhecidas para que intervenções no combate a novas infecções possam ser implementadas. Objetivo: Realizar um levantamento epidemiológico de HIV / AIDS no município de Parnaíba-PI, no período de 1990-2018. Métodos: Estudo descritivo, transversal e quantitativo, realizado a partir de dados disponibilizados pelo Departamento de Informática do Sistema Único de Saúde (DATASUS) e pelo Departamento de Doenças de Condições Crônicas e Infecções Sexualmente Transmissíveis, da Secretaria de Vigilância em Saúde, do Ministério da Saúde (DCCI/SVC/MS). Resultados: Foram registrados 372 casos de HIV / AIDS com as maiores prevalências observadas no sexo masculino $(65,9 \%)$, raça/cor parda $(36,8 \%)$, nível fundamental incompleto $(44,9 \%)$, categoria de exposição heterossexual $(34,9 \%)$ e faixa etária entre 20-49 anos (85,2\%). A taxa média de detecção de infecção pelo HIV foi de 9,2/100.000 hab. Outro fator relevante, diz respeito ao número de gestantes com cerca de $12,6 \%$ do total de mulheres infectadas. Foram registrados o total de 114 óbitos por AIDS, com taxa média de mortalidade de 2,8/100.000 hab. e letalidade de 30,6\%. Conclusão: O estudo permitiu discutir sobre as variáveis epidemiológicas e a necessidade de avanços em políticas públicas específicas, a exemplo das mulheres gestantes e dos homens, além do incentivo ao diagnóstico e ao trabalho permanente em educação e prevenção, para que haja redução da morbimortalidade por esse agravo.

Palavras-Chave: HIV, epidemiologia, AIDS.

\section{ABSTRACT}

Introduction: The HIV/AIDS epidemic is a challenge to global public health and leads to death thousands of people every year. Today it is considered a chronic condition, with important epidemiological changes that need to be known so that interventions to fight new infections can be implemented. Objective: To perform an epidemiological survey of HIV/AIDS in the municipality of Parnaíba-PI, in the period 1990-2018. Methods: Descriptive, cross-sectional and quantitative study, based on data provided by the Department of Informatics of the Unified Health System (DATASUS) and by the Department of Diseases of Chronic Conditions and Sexually Transmitted Infections, of the Secretary of Health Surveillance, of the Ministry of Health (DCCI/SVC/MS). Results: 372 cases of HIV/AIDS were registered with the highest prevalences observed in males $(65.9 \%)$, brown race/color $(36.8 \%)$, incomplete fundamental level $(44.9 \%)$, heterosexual exposure category $(34.9 \%)$ and age group between $20-49$ years $(85.2 \%)$. The average HIV infection detection rate was 9.2/100,000 inhabitants. Another relevant factor concerns the number of pregnant women with about $12.6 \%$ of the total number of infected women. A total of 114 deaths from AIDS were recorded, with an average mortality rate of 2.8/100,000 inhabitants and lethality of 30.6\%. Conclusion: The study allowed to discuss the epidemiological variables and the need for progress on specific public policies, like the pregnant women and men, in addition to encouraging the diagnosis 
and permanent work on education and prevention, so that there is reduction in morbidity and mortality from this injury.

Keywords: HIV, epidemiology, AIDS.

\section{RESUMEN}

Introducción: La epidemia de VIH / SIDA es un desafío para la salud pública en todo el mundo y provoca la muerte de miles de personas cada año. Hoy se considera una condición crónica, con importantes cambios epidemiológicos que deben conocerse para que se puedan implementar intervenciones para combatir nuevas infecciones Objetivo: Realizar una encuesta epidemiológica del VIH/SIDA en la ciudad de Parnaíba-PI, en el período 1990-2018. Métodos: Estudio descriptivo, transversal y cuantitativo, basado en datos proporcionados por el Departamento de Informática del Sistema Único de Salud (DATASUS) y por el Departamento de Enfermedades de Condiciones Crónicas e Infecciones de Transmisión Sexual, del Departamento de Vigilancia Sanitaria, del Ministerio de Salud (DCCI/SVC/MS). Resultados: Se registraron 372 casos de VIH/SIDA con las prevalencias más altas observadas en hombres $(65.9 \%)$, raza/color marrón (36.8\%), nivel fundamental incompleto $(44.9 \%)$, categoría de exposición heterosexual (34.9\%) y grupo de edad entre 20-49 años $(85.2 \%)$. La tasa promedio de detección de infección por VIH fue de 9.2/100,000 habitantes. Outro factor relevante se refiere al número de mujeres embarazadas con aproximadamente el $12.6 \%$ del número total de mujeres infectadas. Se registraron un total de 114 muertes por SIDA, con una tasa de mortalidad promedio de 2.8/100,000 habitantes y letalidad del 30.6\%. Conclusión: El estudio permitió discutir las variables epidemiológicas y la necesidad de avances en políticas públicas específicas, como mujeres embarazadas y hombres, además de alentar el diagnóstico y el trabajo permanente en educación y prevención, a fin de reducir la morbilidad y la mortalidad debido a este agravio.

Palabras clave: VIH, epidemiología, SIDA. 


\section{Introdução}

A Organização Mundial de Saúde (OMS), em 2015, estimou que cerca de 78 milhões de pessoas estariam infectadas pelo Vírus da Imunodeficiência Humana (HIV), e que 39 milhões de homens, mulheres e crianças foram a óbito por alguma complicação causada pela infecção ${ }^{1,2}$. No transcorrer dos anos, estas infecções têm levado enormes sequelas para famílias, comunidades e países, e tornou-se um dos maiores desafios para a saúde pública³.

Em 2014, estimou-se que cerca de 718 mil indivíduos viviam com o HIV no Brasil, no entanto, apenas $80 \%$ tinha conhecimento sobre o seu diagnóstico. Do ano de 2007 até junho de 2018, foram detectados, pelo Ministério da Saúde, 247.795 casos de HIV. Desse total, 42.215 (17\%) localiza-se na Região Nordeste (NE). Em relação à mortalidade por HIV, desde o início da epidemia no ano de 1980 até o final do ano de 2017, os achados identificaram cerca de 327.655 óbitos no Brasil, sendo que, 13,3\% representa os óbitos no $\mathrm{NE}^{4,5}$.

No Brasil, em 2017, foram diagnosticados 42.420 novos casos de HIV e 37.791 casos de AIDS, notificados no SINAN (Sistema de Informação de Agravos de Notificação), e declarados no SIM (Sistema de Informações de Mortalidade), com uma taxa de detecção de 18,3/100.000 hab., totalizando no período de 1980 a junho de 2018, 982.129 casos detectados no país 6 .

Em 2018, segundo a Organização das Nações Unidas (ONU), cerca de aproximadamente 7.000 pessoas foram infectadas pelo HIV diariamente, e que uma pessoa morre a cada 20 segundos por uma doença associada à Síndrome da Imunodeficiência Adquirida (AIDS). A região da África Subsaariana continua sendo a mais atingida, com $60 \%$ das pessoas vivendo com HIV, onde as mulheres representam $58 \%$ deste total ${ }^{3}$.

Pelo Sistema Único de Saúde (SUS) o diagnóstico para o HIV pode ser lizado em Unidades Básicas de Saúde (UBS), assim como no Centro de Testagem Aconselhamento (CTA) para Infecções Sexualmente Transmissíveis (IST) e AIDS, se encontram distribuídos por todo o país7. Tem sido utilizado também o Teste ido (TR) no diagnóstico para HIV, cuja metodologia permite a detecção de 
anticorpos em até 30 minutos e, além disso, possui baixo custo e é altamente sensível, específico, de fácil aplicação e interpretação ${ }^{8}$.

No município de Parnaíba-PI, a Rede de Atenção à Saúde (RAS) conta com CTA/Serviço de Assistência Especializada (SAE), UBS e outros serviços públicos que acolhem pessoas com vulnerabilidade quanto à IST e que realizam o TR e/ou o diagnóstico laboratorial específico para essas infecções. Esse levantamento fez parte das ações do Programa de Educação pelo Trabalho para a Saúde (PET-Saúde) /Interprofissionalidade, Parnaíba-PI, do Ministério da Saúde, que objetiva induzir a reflexão e mudanças na formação profissional, além de identificar as necessidades no serviço de saúde, a fim de trazer propostas de intervenção.

Mediante o exposto e considerando a situação dos portadores de HIV/ AIDS , no Brasil e no mundo, e das consequências que a infecção causa nesses indivíduos, o presente trabalho teve como finalidade realizar um levantamento epidemiológico das pessoas com HIV/AIDS no município de Parnaíba-PI, no período de 1990 a 2018, objetivando contribuir na identificação de variáveis epidemiológicas específicas que careçam de um trabalho de mudança e/ou de novas medidas operacionais, no âmbito do serviço de saúde municipal ofertado, para que se possa alcançar uma melhoria nos índices.

\section{Metodologia}

Tratou-se de um estudo descritivo, transversal e quantitativo, realizado a partir de dados disponibilizados pelo Departamento de Informática do Sistema Único de Saúde (DATASUS) e do Departamento de Doenças de Condições Crônicas e Infecções Sexualmente Transmissíveis, da Secretaria de Vigilância em Saúde, do Ministério da Saúde (DCCI/SVC/MS). As informações contidas no DATASUS são provenientes de três sistemas de informação: Sistema de Informações de Agravos de Jotificação (SINAN), Sistema de Controle de Exames Laboratoriais da Rede cional de Contagem de Linfócitos CD4+/CD8 e Carga Viral (SISCEL) e Sistema de formações de Mortalidade (SIM). Os dados coletados foram tabulados em planilha twares Microsoft Excel, analisados no programa e apresentados como estatística 


\section{ciênncia
pural}

descritiva (média), taxas, números absolutos $(\mathrm{N})$ e frequências relativas (\%), para análise da distribuição das diferentes variáveis. Para o cálculo de taxas foram utilizadas as informações populacionais fornecidos pelo Instituto Brasileiro de Geografia e Estatística (IBGE). Os dados coletados para esse trabalho foram do município de Parnaíba o qual situa-se no extremo Norte do Estado do Piauí e encontra-se localizado à $339 \mathrm{~km}$ da capital, Teresina. Tendo uma área total de 436 km² e uma população estimada para 2019 de 153.078 habitantes.

Para melhor compreensão da situação epidemiológica do HIV/AIDS em Parnaíba-PI, os dados foram analisados, segundo as variáveis: raça/cor (branca, preta, parda, amarela, indígena), sexo (masculino e feminino), nível de aprendizado adquirido (analfabeto, fundamental incompleto ou completo, médio completo e superior completo), ano de diagnóstico, gestantes infectadas pelo HIV, óbitos por causa básica AIDS, categoria de exposição (orientação sexual, usuários de drogas injetáveis, hemofílicos, transfusão de sangue, acidente de trabalho com material biológico e transmissão vertical) e faixa etária. Por critério de inclusão considerou-se todos os casos de HIV/AIDS diagnosticados entre os anos 1990 a 2018. Com exceção da variável gestantes infectadas pelo HIV, onde os dados foram obtidos do DCCI/SVC/MS, todas as outras variáveis tiveram como fonte de informações o DATASUS.

O projeto foi submetido e aprovado no Comitê de Ética e Pesquisa (CEP) da Universidade Federal do Piauí/UFPI , com parecer nº 2.402.713.

\section{Resultados}

Durante quase três décadas, de 1990 a 2018, foram notificados um total de 372 casos de HIV/AIDS. Constatou-se que o ano de 2016 obteve o maior número de registros $(\mathrm{N}=34)$, equivalente a 9,1\% das notificações. O número de casos no sexo asculino foi de 245 (65,9\%), enquanto para o feminino foi de 127 (34,1\%), uma lação de 1,9 homens para cada mulher. Observou-se, também, que na primeira ada (1990-1999) foram detectados 37 casos positivos, sendo praticamente a 
totalidade ( $\mathrm{N}=34 ; 91,9 \%)$ desses no sexo masculino, o que representaram um número 11,3 vezes maior que o constatado para o sexo feminino $(\mathrm{N}=03 ; 8,1 \%)$.

No presente estudo, para efeito de entendimento das diferenças e/ou evolução quantitativas, algumas variáveis tiveram dentro do período do levantamento, o número de casos da primeira década comparado à última (2009-2018). Apesar do aumento do número total de casos diagnosticados $(\mathrm{N}=220)$ na última década, foi possível detectar que, comparativamente a primeira, a correlação entre os gêneros diminuiu para 1,8 vezes, onde se observou no sexo masculino 142 casos $(64,5 \%)$ e no feminino 78 casos $(35,5 \%)$ (tabela 1$)$. A taxa média de detecção de infecção pelo HIV para o período foi de 9,2/100.000 hab., sendo o ano de 2016 o que apresentou a maior taxa de infecção com 22,6/100.000 hab.

Tabela 1. Distribuição do número de casos de HIV / AIDS, por ano de diagnóstico e gênero, Parnaíba-PI, 1990-2018.

\begin{tabular}{|c|c|c|c|}
\hline Ano & Masculino & Feminino & Total \\
\hline 1990 & 01 & 00 & 01 \\
\hline 1991 & 02 & 00 & 02 \\
\hline 1992 & 02 & 00 & 02 \\
\hline 1993 & 01 & 00 & 01 \\
\hline 1994 & 13 & 00 & 13 \\
\hline 1995 & 03 & 00 & 03 \\
\hline 1996 & 03 & 01 & 04 \\
\hline 1997 & 03 & 00 & 03 \\
\hline 1998 & 02 & 00 & 02 \\
\hline 1999 & 04 & 02 & 06 \\
\hline 2000 & 05 & 03 & 08 \\
\hline 2001 & 05 & 03 & 08 \\
\hline 2002 & 06 & 06 & 12 \\
\hline 2003 & 05 & 02 & 07 \\
\hline 2004 & 15 & 02 & 17 \\
\hline 2005 & 08 & 05 & 13 \\
\hline 2006 & 06 & 09 & 15 \\
\hline 2007 & 06 & 10 & 16 \\
\hline 2008 & 13 & 06 & 19 \\
\hline 2009 & 19 & 08 & 27 \\
\hline 2010 & 06 & 06 & 12 \\
\hline 2011 & 16 & 07 & 23 \\
\hline 2012 & 15 & 05 & 20 \\
\hline 2013 & 15 & 11 & 26 \\
\hline 2014 & 13 & 09 & 22 \\
\hline
\end{tabular}




\begin{tabular}{lccc}
\hline 2015 & 17 & 08 & 25 \\
2016 & 22 & 12 & 34 \\
2017 & 04 & 05 & 09 \\
2018 & 15 & 07 & 22 \\
Total & $\mathbf{2 4 5}$ & $\mathbf{1 2 7}$ & $\mathbf{3 7 2}$ \\
\hline
\end{tabular}

Em relação a raça/cor, pode-se observar que, do número total de casos $(\mathrm{N}=372)$, a maior prevalência ocorreu na população parda $(\mathrm{N}=137 ; 36,8 \%)$, seguida da branca $(\mathrm{N}=27 ; 7,2 \%)$. A população da raça/cor preta $(\mathrm{N}=9 ; 2,4 \%)$ e amarela $(\mathrm{N}=1$; $0,2 \%$ ) demonstraram menores frequências. Não houve registro de HIV/ AIDS para a indígena, bem como os classificados como ignorados somaram 198 casos $(53,2 \%)$. Constatou-se também que, nos anos de 2015 e 2017, 100\% dos casos foram de pessoas pardas.

Sobre o nível de aprendizado adquirido, observou-se 185 registros onde a categoria fundamental incompleto obteve o maior número de casos $(\mathrm{N}=83 ; 44,9 \%)$ de HIV/AIDS do total. As menores frequências foram verificadas nas categorias analfabeto e superior completo, sendo $\mathrm{N}=14(7,6 \%)$ e $\mathrm{N}=18$ (9,7\%), respectivamente (tabela 2).

Tabela 2. Distribuição dos casos de HIV/ AIDS, segundo nível de aprendizado adquirido, Parnaíba-PI, 1990-2018.

\begin{tabular}{ccc}
\hline Nível de Aprendizado Adquirido & $\mathbf{n}$ & $\mathbf{\%}$ \\
Analfabeto & 14 & 7,6 \\
Fundamental incompleto & 83 & 44,9 \\
Fundamental completo & 32 & 17,3 \\
Médio completo & 35 & 18,9 \\
Superior completo & 18 & 9,7 \\
Não se aplica & 03 & 1,6 \\
Total & $\mathbf{1 8 5}$ & $\mathbf{1 0 0}$ \\
\hline
\end{tabular}

Outro fator relevante diz respeito ao número de gestantes infectadas pelo HIV. s 372 casos de HIV/AIDS, 16 correspondem às gestantes, o que equivale a 12,6\% total de mulheres infectadas (tabela 3). Não houve casos registrados em alguns s, bem como observou-se 01 caso em 2011. Ressalta-se que o sistema de 
informação (DCCI/SVC/MS) para essa variável, não disponibilizou a estratificação quantitativa dos casos entre os anos de 1990-2006.

Tabela 3. Distribuição do número de gestantes infectadas pelo HIV, por ano do parto, Parnaíba-PI, 1990-2018.

\begin{tabular}{cccc}
\hline Ano & Casos HIV & $\begin{array}{c}\text { Mulheres com } \\
\text { HIV }\end{array}$ & $\begin{array}{c}\text { Gestantes com } \\
\text { HIV }\end{array}$ \\
$\mathbf{1 9 9 0 - 2 0 0 6}$ & 117 & 33 & 08 \\
$\mathbf{2 0 0 7}$ & 16 & 10 & 02 \\
$\mathbf{2 0 0 8}$ & 19 & 06 & 00 \\
$\mathbf{2 0 0 9}$ & 27 & 08 & 00 \\
$\mathbf{2 0 1 0}$ & 12 & 06 & 00 \\
$\mathbf{2 0 1 1}$ & 23 & 07 & 01 \\
$\mathbf{2 0 1 2}$ & 20 & 05 & 00 \\
$\mathbf{2 0 1 3}$ & 26 & 11 & 00 \\
$\mathbf{2 0 1 4}$ & 22 & 09 & 00 \\
$\mathbf{2 0 1 5}$ & 25 & 08 & 03 \\
$\mathbf{2 0 1 6}$ & 34 & 12 & 00 \\
$\mathbf{2 0 1 7}$ & 09 & 05 & 00 \\
$\mathbf{2 0 1 8}$ & 22 & 07 & 02 \\
Total & $\mathbf{3 7 2}$ & $\mathbf{1 2 7}$ & $\mathbf{1 6}$ \\
\hline
\end{tabular}

Acerca dos óbitos, por causa básica AIDS, foram contabilizados 114 registros. Vale destacar que na primeira década (1990-1999), ocorreram 11 mortes (9,6\%) do total e, comparativamente a última década do período investigado (2009-2018), houve um aumento de 6,8 vezes onde se observou 75 mortes $(65,8 \%)$. O ano de 2009 apresentou a maior frequência $(\mathrm{N}=12 ; 10,5 \%)$, seguido dos anos de 2016 e 2018 onde ocorreram, em cada um deles, 11 óbitos (9,6\%) (tabela 4). A taxa de letalidade para o período e a taxa média de mortalidade por AIDS foram de 30,6\% e 2,8/100.000 hab., respectivamente. 
Tabela 4. Distribuição do número de óbitos por causa básica AIDS, por ano do óbito, Parnaíba-PI, 1990-2018.

\begin{tabular}{|c|c|c|}
\hline \multirow[t]{2}{*}{ Ano } & \multicolumn{2}{|c|}{ Óbitos por AIDS } \\
\hline & (n) & $\%$ \\
\hline 1990 & 00 & 00 \\
\hline 1991 & 00 & 00 \\
\hline 1992 & 02 & 1,8 \\
\hline 1993 & 00 & 00 \\
\hline 1994 & 03 & 2,6 \\
\hline 1995 & 02 & 1,8 \\
\hline 1996 & 03 & 2,6 \\
\hline 1997 & 00 & 00 \\
\hline 1998 & 01 & 0,9 \\
\hline 1999 & 00 & 00 \\
\hline 2000 & 03 & 2,6 \\
\hline 2001 & 02 & 1,8 \\
\hline 2002 & 03 & 2,6 \\
\hline 2003 & 04 & 3,5 \\
\hline 2004 & 03 & 2,6 \\
\hline 2005 & 01 & 0,9 \\
\hline 2006 & 04 & 3,5 \\
\hline 2007 & 03 & 2,6 \\
\hline 2008 & 05 & 4,4 \\
\hline 2009 & 12 & 10,5 \\
\hline 2010 & 03 & 2,6 \\
\hline 2011 & 07 & 6,1 \\
\hline 2012 & 09 & 7,9 \\
\hline 2013 & 05 & 4,4 \\
\hline 2014 & 05 & 4,4 \\
\hline 2015 & 05 & 4,4 \\
\hline 2016 & 11 & 9,6 \\
\hline 2017 & 07 & 6,1 \\
\hline 2018 & 11 & 9,6 \\
\hline Total & 114 & 100 \\
\hline
\end{tabular}


A análise da distribuição dos casos de HIV/AIDS, segundo categoria de exposição hierarquizada, demonstrou que a transmissão sexual é predominante ( $\mathrm{N}=180 ; 48,4 \%)$ com maior número de casos na orientação sexual heterossexual $(\mathrm{N}=130 ; 34,9 \%)$, seguida da homossexual e bissexual que, juntas, somaram 50 casos $(13,4 \%)$. As demais categorias, comparativamente, não apresentaram dados relevantes. Pode-se observar que existe um número considerável de dados ignorados $(\mathrm{N}=179 ; 48,1 \%)$, o que poderia alterar as frequências em relação as diferentes categorias/subcategorias.

Tabela 5. Distribuição dos casos de HIV/AIDS, segundo categoria de exposição hierarquizada, Parnaíba-PI, 1990-2018.

\begin{tabular}{cccc}
\hline Categoria de Exposição & Subcategoria & $\mathbf{N}$ & $\%$ \\
& Homossexual & 32 & 8,6 \\
Orientação sexual & Bissexual & 18 & 4,8 \\
& Heterossexual & 130 & 34,9 \\
Usuários de Drogas & & 08 & 2,1 \\
Injetáveis & & - & - \\
Hemofílico & & - & - \\
Transfusão & & - & - \\
Acidente com & & 05 & 1,3 \\
Material Biológico & & 179 & 48,1 \\
Transmissão Vertical & & 372 & $\mathbf{1 0 0}$ \\
Ignorado & & & \\
Total & & & \\
\hline
\end{tabular}

Por fim, acerca da variável faixa etária, do número total de casos de HIV/ AIDS $(\mathrm{N}=327)$, constatou-se que a maioria se concentrou entre $20-49$ anos $(\mathrm{N}=317 ; 85,2 \%)$. casos com idade $<1$ ano $(\mathrm{N}=03 ; 0,8 \%)$ e aqueles com idade $\geq 80$ anos $(\mathrm{N}=01 ; 0,2 \%)$ resentaram as menores frequências. Verificou-se, também, um número de casos vante em outras faixas etárias: 01-09 anos ( $\mathrm{N}=05 ; 1,3 \%), 10-19$ anos $(\mathrm{N}=09 ; 2,4 \%)$ -79 anos $(\mathrm{N}=37 ; 9,9 \%)$. 


\section{Discussão}

No período compreendido entre os anos de 1990 a 2018, os dados do município de Parnaíba-PI seguiram o padrão, mundial e brasileiro, em relação ao maior número de casos de HIV/AIDS no sexo masculino (tabela1). O boletim epidemiológico de HIV/AIDS - 2018, do Departamento de Vigilância, Prevenção e Controle das Infecções Sexualmente Transmissíveis, do HIV/AIDS e das Hepatites Virais, da Secretaria de Vigilância em Saúde, do Ministério da Saúde (DIAHV/SVS/MS) traz uma análise entre os anos de 2007 a 2018 onde há predominância de notificações no sexo masculino ${ }^{6}$.

Recentemente, os resultados de um estudo realizado na cidade de Novo Hamburgo-RS, mostrou que o sexo masculino representou a maioria dos casos de HIV / AIDS, entre 2007 a 2018. Ressaltam ainda que, o número de diagnóstico de HIV aumentaram ao longo dos anos e os casos de AIDS reduziram, o que pode ser reflexo da ampliação da testagem e maior diagnóstico e tratamento em tempo oportuno. No entanto, o aumento de casos HIV evidencia a importância do município em trabalhar mais com prevenção e ações educativas ${ }^{9}$. De acordo com o portal da Prefeitura Municipal de Parnaíba-PI (2015), o maior número de notificações ocorridas no ano de 2016 (N=34) em Parnaíba-PI, pode ser atribuída a descentralização da testagem, onde os testes rápidos passaram a ser realizados também nas unidades básicas de saúde do município ${ }^{10}$.

No presente levantamento, apesar do número de casos no sexo masculino ter sido superior ao feminino na primeira (1990-1999) e na última (2009-2018) décadas do período desse levantamento, observou-se que houve uma feminização da infecção, com o incremento, na última década, no número de casos diagnosticados e HIV/AIDS de 26 vezes para o sexo feminino, enquanto o masculino foi de 4,7 vezes (Tabela 1). Um estudo realizado, durante o período de 1996 a 2016, com 457 pacientes no estado de Rondônia, também evidenciou o aumento do número de casos no sexo feminino ao longo dos anos, onde a prevalência de infecção pelo HIV foi de $65 \%$ e $35 \%$ no masculino ${ }^{11}$. 
Já em relação a raça/cor, de acordo com dados do Instituto Brasileiro de Geografia e estatística (IBGE) de 2015, a população parnaibana tem uma maior prevalência somada de pardos e negros ${ }^{12}$, corroborando com o resultado desse trabalho que evidenciou maior notificação de pessoas pardas. Semelhante aos nossos achados, um estudo realizado na cidade de Porto Alegre-RS teve a prevalência da população não branca ${ }^{13}$. No entanto, um levantamento realizado em munícipios do Brasil, entre os anos de 2012 a 2016, ressalta a questão da subnotificação em cada um dos estratos, sendo de $24,3 \%$ na raça indígena, 30,1\% na negra (preta e parda), $31,1 \%$ na branca, $43,4 \%$ entre a raça amarela e $74,1 \%$ entre os casos com essa informação ignorada ${ }^{14}$. Nesse presente estudo, a informação acerca da raça/cor autodeclarada somente foi possível para 129 casos de HIV / AIDS, uma vez que 22,3\% $(\mathrm{N}=50)$ tiveram esse dado ignorado nos registros.

O baixo nível de aprendizado foi um achado desse levantamento e converge com os dados encontrados no boletim epidemiológico de HIV/AIDS-2018, onde destacou-se a concentração dos casos de HIV/AIDS em pessoas com escolaridade classificada como ensino fundamental incompleto, entre os anos de 1980-20186. Estudos que abordam a variável escolaridade evidenciam que existe uma alta proporção de indivíduos com baixa escolaridade e a maioria exercendo ocupações diversas concomitante a prática estudantil. Estes achados estão em consonância com a literatura mundial que evidencia modificações no padrão de transmissão do HIV, em decorrência do baixo nível de formação escolar, sugerindo a possível relação entre pauperização populacional e infecção ou doença. A disseminação do HIV junto às camadas mais pobres e com baixo nível de escolaridade aponta maior susceptibilidade deste grupo, possivelmente pela falta de informações e menor acesso aos meios de prevenção ${ }^{15}$.

Corroborando com os achados desse trabalho, uma pesquisa realizada no estado do Piauí sobre o uso de antirretrovirais em indivíduos com HIV/AIDS, em Hospital de referência em Infectologia, pode-se observar a baixa escolaridade ${ }^{16}$. sim, ao pensar em estratégias de prevenção às IST no município de Parnaíba, na-se relevante levar em consideração as vulnerabilidades sociais do público, o aqueles que já convivem com a infecção, quanto aos saudáveis. 
Ao avaliar as notificações de HIV/AIDS em gestantes no município de Parnaíba, pode-se mais uma vez inferir que a oferta dos testes rápidos ocorrida efetivamente nos anos de 2015 e 2016, propiciou maior detecção ou melhora no diagnóstico do agravo. Destaca-se que por meio da Estratégia da Rede Cegonha, diversas ações contribuíram para melhor detecção de casos de IST em gestantes em todo o país, sendo as principais, a ampliação da oferta de testes rápidos de HIV e sífilis, o aconselhamento em IST/AIDS e a reorganização dos serviços e processos de trabalho'7. Em relação a gestante portadora de HIV/AIDS, observa-se que a notificação deve ocorrer logo que for diagnosticada a infecção pelo vírus, de preferência no primeiro trimestre, após a inserção dos dados referentes ao parto. No entanto, a não realização da sorologia para HIV no início do pré-natal ou o não recebimento do resultado em tempo hábil, prejudica a notificação oportuna do caso e o início das medidas preventivas preconizadas ${ }^{18}$.

A mortalidade por AIDS na cidade de Parnaíba (2,8/100.000 hab.) pode ser remetida, ainda que parcialmente, as diferentes doenças oportunistas. Sendo esta consequência bastante comum aos estigmas criados pela sociedade, levando o indivíduo ao acesso tardio para diagnóstico e tratamento da infecção pelo HIV. A grande maioria dos pacientes não retornam para saber o resultado do seu teste ou após o resultado não aderem ao tratamento, pela desigualdade e discriminação encontrada na prestação de serviços de tratamento, e esses elementos são de extrema relevância para a mortalidade pela doença ${ }^{19,20}$. Recentes publicações evidenciam uma pequena redução da mortalidade por AIDS, de 2000 a 2014, no Brasil. Todavia, existem relevantes diferenças por região. Os dados apontaram a redução desse índice para Região Sudeste e, contrariamente, um aumento no índice de mortalidade para as Regiões Nordeste e Norte. Já as regiões Sul e Centro-Oeste encontram-se estáveis, com discreta redução. Os índices mais elevados foram registrados no Rio de Janeiro, em 2000, e no Rio Grande do Sul em 201421,22.

Acerca dos casos de HIV/ AIDS, segundo categoria de exposição, a orientação xual representou 48,3\% dos casos, sendo a maioria destes $(34,9 \%)$ declarados erossexuais (tabela 5). Um estudo realizado em um CTA da cidade de Fortalezasobre o uso de preservativo nas relações sexuais, destacou que o motivo mais 
prevalente para o não uso, era "o não gostar", em que ambos os sexos citaram o não uso em parcerias eventuais ${ }^{7}$. Portanto, conhecer características comportamentais dos usuários pode auxiliar na elaboração de ações para prevenir novos casos. Um estudo realizado no estado do Rio Grande do Norte no período 1980-2013 com 4.666 casos de AIDS, constatou, assim como os resultados desse trabalho, que os heterossexuais eram a categoria de exposição mais frequente $(39,1 \%)$, seguida da homossexual $(15,1 \%)^{23}$.

Outro levantamento realizado no estado de Minas Gerias, entre 2007 e 2016, verificaram que a transmissão sexual era predominante, sendo 50,3\% dos casos de orientação heterossexual e $22,8 \%$ de homossexuais ${ }^{24}$. Corroborando com os achados desse levantamento, o boletim epidemiológico da Secretaria de Vigilância em Saúde/Ministério da Saúde-2018, sobre casos de infecção pelo HIV/AIDS no Brasil entre 2007 a junho de 2018, também constatou que a transmissão sexual é majoritária, sendo $48,6 \%$ dos casos decorrentes de exposição heterossexual e 29,3\% homossexual ${ }^{6}$. Os dados expostos apontam para o fenômeno da heterossexualização da infecção pelo HIV.

Todas as demais variáveis exploradas nesse levantamento tiveram entre os indivíduos mais acometidos aqueles com idade entre 20-49 anos (85,2\%). Intervalo de idade similar ${ }^{6,23}$ ou próximos, tais como (20-59 anos) ${ }^{9,24}$ e (33-45 anos) ${ }^{11}$, foram demonstrados como as faixas etárias mais prevalentes nesses estudos epidemiológicos sobre HIV/AIDS. Desde 2005, o Plano Estratégico do Programa Nacional de DST e AIDS já sinalizava alguns aspectos relevantes sobre o tema, ressaltando a faixa etária de 25-39 anos como sendo a mais afetada pela infecção e aumentando progressivamente a ocorrência de casos no sexo feminino em idade reprodutiva. Outro dado relevante apresentado, diz respeito ao maior número de casos de HIV / AIDS entre 15-54 anos, que pode ser remetido a vida sexualmente ativa 90\% dos brasileiros nessa faixa etária, os quais mantêm mais de 10 parceiros xuais na vida. Porém, entre essa parcela da população, $96 \%$ tem conhecimento que so do preservativo protege da transmissão sexual, o que leva a necessidade efetiva uma mudança comportamental ${ }^{25}$. 


\section{Conclusões}

O presente estudo permitiu refletir acerca das diferentes variáveis epidemiológicas nos indivíduos portadores de HIV / AIDS no município de ParnaíbaPI. Evidenciou-se que o perfil segue semelhante ao brasileiro, sobretudo no que se refere ao nível de aprendizagem e raça/cor, numa relação direta, já bem conhecida e explorada, com a vulnerabilidade socioeconômica dessa parcela da população.

Ainda, esse levantamento permitiu oportunizar aos profissionais do serviço de saúde municipal, e todos os outros envolvidos, um olhar ampliado da assistência às pessoas convivendo com HIV / AIDS. Percebeu-se, então, a necessidade de avanços nas políticas de saúde voltadas para esse público, ampliando o trabalho contínuo de testagem/diagnóstico, bem como de educação e prevenção, considerando as individualidades principalmente para os grupos mais acometidos, como os homens, e para $\mathrm{o}$ acolhimento $\mathrm{e}$ trabalho assistencialista às gestantes infectadas, principalmente devido a feminização da infecção ao longo dos anos.

Somente assim poderemos alcançar uma melhoria/diminuição nos indicadores, a exemplo da taxa de detecção de infecção pelo HIV em 2016 (22,6/100.000 hab) no município de Parnaíba-PI que foi superior, nesse mesmo ano, a nacional (18,8/100.000 hab.), segundo dados do últimos Boletim Epidemiológico HIV / AIDS da Secretaria de Vigilância em Saúde/Ministério da Saúde.

\section{Financiamento}

Este estudo fez parte das ações do Programa de Educação pelo Trabalho para a Saúde (PET-Saúde)/Interprofissionalidade, Parnaíba-PI, e foi financiado pelo Ministério da Saúde, edital n. 10 de 23 de julho de 2018. 


\section{Referências}

1. Word Health Organization. World health statistics 2015. Genebra (Suíça): 2015.

2. Sousa GA, Pessoa RS, Souza Filho MCM, Oliveira DFM, Medeiros LL, Souza DLB, Costa I CC. Factors associated with mortality in hepatitis c patients. Rev. Ciênc. Plural [Internet]. 2017 Abril [citado 6 de abril de 2020];2(3):45-8. Disponível em: https://periodicos.ufrn.br/rcp/article/view/11624

3. Programa Conjunto das Nações Unidas sobre HIV/Aids. A ONU e a resposta à AIDS no Brasil. Brasília-DF: 2013.

4. Martins TA, Kerr L, Kendall C, Mota RMS. Cenário Epidemiológico da Infecção pelo HIV e AIDS no Mundo. Rev. Fisioter S Fun [Internet]. 2014 Jun [citado em 10 de fevereiro de 2020]; 3(1):4-7. Disponível em: http:/ / periodicos.ufc.br/fisioterapiaesaudefuncional/article/view/20575

5. Lins MEVS, Jesus JB, Oliveira JF, Rêgo GG, Matos AVM, Wanderley NB, et al. Perfil epidemiológico de óbitos por HIV/AIDS na região nordeste do Brasil utilizando dados do sistema de informação de saúde do DATASUS/Epidemiological profile of HIV/AIDS deaths in northeastern Brazil using data from the DATASUS health information system. Rev. Braz. J of Health Review [Internet]. 2019 Maio [citado em 10 de fevereiro de 2020]; 2(4):2965-73. Disponível em: http:/ / www.brazilianjournals.com/index.php/BJHR/article/view/2048

6. Ministério da Saúde. Secretaria de Vigilância em Saúde Departamento de Vigilância, Prevenção e Controle das Infecções Sexualmente Transmissíveis, do HIV/Aids e das Hepatites Virais. Boletim Epidemiológico de HIV/AIDS 2018 (DIAHV) - Brasília, DF; 2018.

7. Sousa Nogueira FJ, Callou Filho CR, Mesquita CAM, Souza ES, Saraiva AKM. Caracterização dos usuários atendidos em um centro de testagem e aconselhamento em infecções relacionadas ao sexo. Rev. Saúde e Pesquisa [Internet]. 2017 Set [citado em 17 de janeiro de 2020]; 10(2):243-50. Disponível em: https://periodicos.unicesumar.edu.br/index.php/saudpesq/article/view/5861

8. Oliveira JAS, Afonso TM. O Teste Rápido para o Diagnostico de HIV na Atenção Primária à Saúde e a importância da atuação do Enfermeiro. Congresso Internac. de Enferm. [Internet]. 2017 Agos [citado em 3 de fevereiro de 2020];1(1). Disponível em: https://eventos.set.edu.br/index.php/cie/article/view/5714

Kuhn BJB. Perfil sociodemográfico dos casos de HIV e AIDS de residentes de Novo mburgo-RS, diagnosticados entre 2007 e 2018. [trabalho de conclusão de pecialização]. Porto Alegre: Universidade Federal do Rio Grande do Sul; 2019. 
10. Portal da Prefeitura de Parnaíba [https://parnaiba.pi.gov.br/phb/]. Unidades básicas de Saúde expandem realização de testes rápidos de HIV e Sífilis. [acesso em 05 de novembro de 2019]. Disponível em: https://parnaiba.pi.gov.br/phb/category/ultimas-noticias/

11. Imamura KB, Faria MPR, Toni JCV. Perfil epidemiolódicos de pacientes com hiv/aids, no período de 1996 até 2016 no município de vilhena-ro. Rev. InterSaúde [Internet]. 2019 Set [citado em 11 de março de 2020]; 1(1):2-21. Disponível em: http://revista.fundacaojau.edu.br:8078/journal/index.php/revista_intersaude/arti cle/view/107

12. Instituto Brasileiro de Geografia e Estatística [https://www.ibge.gov.br/]. Características gerais da população [acesso em 10 de novembro de 2019]. Disponível em: https://www.ibge.gov.br/cidades-e-estados/pi/parnaiba.html

13. Brand ÉM, Rossetto M, Silva Calvo K, Winkler GB, Silva DAR, Hentges B, et al. Factors associated with directly observed treatment in tuberculosis/HIV coinfection cases in Porto Alegre, 2009-2013: A retrospective cohort. Rev. PloS one [Internet]. 2019 Out [citado em 11 de março de 2020]; 14(10). Disponível em: https:/ /journals.plos.org/plosone/article?id=10.1371/journal.pone.0222786

14. Coelho RDA. Estudo da distribuição da subnotificação do HIV/aids no Brasil, 2012 a 2016. [dissertação]. Brasília: Universidade de Brasília; 2019.

15. Pereira BDS, Costa MCO, Amaral MTR, Costa HSd, Silva CALD, Sampaio VS. Fatores associados à infecção pelo HIV/AIDS entre adolescentes e adultos jovens matriculados em Centro de Testagem e Aconselhamento no Estado da Bahia, Brasil. Rev. Ciênc. \& Saúde Colet. 2014;19:747-58.

16. Santos RR. Conhecimento e práticas dos profissionais de saúde dasmaternidades públicas de Teresina, Piauí, no manejo da sífilis na gestação e congênita [dissertação]. Rio de Janeiro: Escola Nacional de Saúde Pública; 2015.

17. Silva APD, Corrêa CM, Barbosa JAG, Borges CM, Souza MCMRd. Aconselhamento em HIV/AIDS e sífilis às gestantes na atenção primária. Rev. enferm UFPE on line [Internet]. 2018 Jul [citado em 17 de janeiro de 2020]; 12(7):19629.

Disponível

em: https:/ / periodicos.ufpe.br/revistas/revistaenfermagem/article/view/236251/294

Meirelles MQB, Lopes AKB, Lima KC. Vigilância epidemiológica de HIV/Aids gestantes: uma avaliação acerca da qualidade da informação disponível. Rev. nam de Salud Pública [Internet]. 2016 Dez [citado em 6 de março de 2020]; (6):427-434. Disponível em: ps:/ / www.scielosp.org/article/rpsp/2016.v40n6/427-434/ 
19. Germano FN, Silva TMGd, Mendoza-Sassi R, Martínez AMB. Alta prevalência de usuários que não retornam ao Centro de Testagem e Aconselhamento (CTA) para o conhecimento do seu status sorológico: Rio Grande, RS, Brasil. Rev. Ciênc. \& Saúde Colet. 2008;13(3):1033-40.

20. Lelis RT, Soares GB, Garbin AJI, Garbin CAS. Discriminação vivenciada por pessoas que vivem com hiv/aids nos serviços de saúde: um estudo qualitativo. Rev. Ciênc. Plural [Internet]. 2017 Abril [citado 6 de abril de 2020];2(3):17-9. Disponível em: https://periodicos.ufrn.br/rcp/article/view/10986

21. Grangeiro A, Escuder MML, Castilho EAD. A epidemia de AIDS no Brasil e as desigualdades regionais e de oferta de serviço. Rev. Cader. de Saúde Púb. [Internet]. 2010 Dez [citado em 7 de março de 2020];26(12):2355-67. Disponível em: https://www.scielosp.org/article/csp/2010.v26n12/2355-2367/pt/

22. Guimarães MDC, Carneiro M, Abreu DMXd, França EB. Mortalidade por HIV / Aids no Brasil, 2000-2015: motivos para preocupação? Rev. bras. epidemiol. 2017;20:182-90.

23. Silva ITS, Silva DC, Góes Salvetti M, Torres GV, da Silva RAR, Souza NL. Perfil dos casos de síndrome da imunodeficiência adquirida em um estado do Nordeste do Brasil. Rev. de Enferm. da UFSM [Internet]. 2014 Dez [citado em 17 de janeiro de 2020];4(4):727-38. Disponível em: https:// periodicos.ufsm.br/index.php/reufsm/article/view/15207

24. Castro SDS, Scatena LM, Miranzi A, Miranzi Neto A, Nunes AA. Temporal trend of HIV/AIDS cases in the state of Minas Gerais, Brazil, 2007-2016. Epidemiol. Serv. Saúde. 2020;29.

25. Programa Nacional de DST/Aids. Plano Estratégico Programa de DST e Aids 2005 [Internet]. 2005 [acesso em 2020 jun 20]. Disponível em: https://bvsms.saude.gov.br/bvs/publicacoes/plano_estrategico.pdf 